RHIC/AP/89

\title{
SIXTRACK Postprocessing for TEAPOT Output
}

\author{
Wolfram Fischer
}

March 1, 1996

\section{Introduction}

Turn-by-turn tracking data hold a large amount of information that can help to understand the nonlinear motion. In addition to the present postprocessing tools for TEAPOT [1] the SIXTRACK [2] postprocessor is now available for TEAPOT turn-by-turn output through a conversion program. Besides other features this allows an easy Lyapunov exponent analysis of the particle motion, necessary to decide if it is regular of chaotic. All programs needed for conversion and analysis are in directory

owl: / rap/lattice_tools/teapot/teasix

In this directory a number of subdirectories exist:

bin executables

example an example of TEAPOT to SIXTRACK conversion

and SIXTRACK postprocessing

sixpost source code for the conversion program

and additional SIXTRACK postprocessing programs

sixtrack SIXTRACK source code (scalar version)

containing the main postprocessor

\section{Transforming TEAPOT Output into SIXTRACK Postprocessing Input}

To transform the TEAPOT turn-by-turn output on file unit 8 into SIXTRACK postprocessing input run

owl: /rap/lattice_tools/teapot/teasix/bin/tpot2six

The program will ask for the phase space dimension. The turn-by-turn coordinates of the particles in fort. 8 will be transfered into file units 90 (1st and 2nd particle), 89 (3rd and 4th particle) etc. in the SIXTRACK format. To perform a Lyapunov analysis the initial particle distances of each pair must be small $\left(10^{-5}\right.$ to $10^{-8} \mathrm{~mm}$ was found to be a good choice for all practical purposes [3]). 


\section{Data Analysis with SIXTRACK}

The SIXTRACK postprocessing parameters must be given in file unit 3 (cf. the example, for details Ref. [2]). The SIXTRACK postprocessor runs with

owl: /rap/lattice_tools/teapot/teasix/bin/six

Depending on the parameters given in unit 3, various output files can be produced. A summary of the postprocessing can be found in fort.6. If graphical output is chosen a postscript file fort. 20 will be created. With the SIXTRACK produced output some further analysis is possible with programs in the bin directory:

tune computes the tune dependent on amplitude or momentum (by M. Giovannozzi)

sixpaw prepares a file from the data in the fort.90-x files that can be read and viewed with paw++

sixpost takes the fort.90-x files and computes the phase advance tune averaged over some turns

setvzero sets the vertical components of the pair coordinates zero, was made to examine the Lyapunov behavior for motion with $z \approx 0$

findxmax finds the maximum horizontal position in the SIXTRACK binary files fort.90-x

Normally, three beta function per degree of freedom (thus coupling included) are contained in the SIXTRACK binary files fort.90-x. Since the conversion program cannot provide these beta functions some results have to be used with care (e.g. smear and nonlinear invariants).

\section{References}

[1] L. Schachinger and R. Talman, "Manual for the program TEAPOT, noninteractive FORTRAN version" (1995).

[2] F. Schmidt, "SIXTRACK, version 1.1, single particle tracking code treating transverse motion with synchrotron oscillations in a symplectic manner", CERN SL/90-52 (AP) (1990). http://hpariel.cern.ch/frs/Documentation/six.ps

[3] F. Schmidt and M. Giovannozzi, private communication. 